\title{
HIGH EFFICIENT SYNCHRONIZATION-ON-DEMAND PROTOCOL OF IEEE802.15.4 WIRELESS SESNOR NETWORK FOR CONSTRUCTION MONITORING
}

\author{
Ji-De Huang ${ }^{1}$, Wen-Jong $\mathrm{Wu}^{2}$, Jen-Yu Han ${ }^{3}$, Hui-Ping Tserng ${ }^{3}$, and Chih-Ting Lin $^{4} *$ \\ ${ }^{1}$ Institute of Applied Mechanics, National Taiwan University, Taipei, Taiwan \\ ${ }^{2}$ Department of Engineering Science and Ocean Engineering, National Taiwan University, Taipei, Taiwan \\ ${ }^{3}$ Department of Civil Engineering, National Taiwan University, Taipei, Taiwan \\ ${ }^{4}$ Graduate Institute of Electronics Engineering, National Taiwan University, Taipei, Taiwan \\ *Corresponding author (timlin@cc.ee.ntu.edu.tw)
}

\begin{abstract}
The Wireless Sensor Network (WSN) technology has been employed in the field of civil engineering especially in structural monitoring and construction safety. To be used in construction safety, the time-synchronization within the sensor network is required to obtain important correlations between events, such as the prevention of equipment collisions. In this work, a high efficient time synchronization method is developed for the ultra-precise localization system to prevent collision accident in construction sites. Without interfering the operation of the whole sensor network, the developed protocol can locally synchronize several nodes within 10ms. By measuring the time uncertainty information through the time stamping, wireless sensor nodes can be synchronized with only two transmitted packages from the beacon or specific nodes. Based on this proposed approach, 0.8 micro-seconds synchronization error was experimentally achieved on an IEEE802.15.4 platform. By employing the developed method, the localization system is implemented and experimentally examined to have the accuracy within several $\mathrm{cm}$ in the range of $2 \mathrm{~m}$. This system can be used to prevent the collision between construction equipments. Therefore, this work demonstrated a simple and efficient approach to obtain good accuracy, low communication overhead, and robust synchronization for most wireless sensor network platforms to be used in automated monitoring in constructions.
\end{abstract}

Keywords: Wireless sensor network, synchronization, ultrasonic localization, Anti-collision system

\section{Introduction}

Because of dynamical and complicated characteristics in civil construction sites, workers and robotic equipments suffer from the potential of collisions [1-3]. To improve the safety of construction sites, strict regulations of field operations are required in construction sites. However, unpredictable collision-accidents happen due to human errors or incaution. As a consequence, several techniques have been proposed to prevent these accidents [2,4]. Among various technologies, the wireless sensor network has drained attentions because it is low-cost, flexible, and easy to be implemented in construction sites [5].

Wireless sensor network (WSN) has become an emerging research field because of its promising potential in different applications and the advancement of integrated circuit technologies [6]. In detail, it consists of hundreds of wireless communication nodes with sensing capabilities to collect the data of interests. To obtain consistency and efficiency between nodes, the coordinated interaction within the network is required [7]. To coordinate sensor node activities, time synchronization is one of the most important feature should be considered in WSN system. Due to resource limitations and decentralized characteristics of WSN, however, time synchronization consideration in WSN is different from wired communication systems [8-9]. Therefore, several protocols, i.e. reference broadcast synchronization (RBS), timing-sync mechanism for sensor networks (TPSN), and 
flooding time synchronization protocol (FTSP), for time synchronization have been proposed and implemented to overcome limitations posed by limited resources in each node [10-11].

Since most of WSN systems have employed IEEE 802.15.4 as their physical (PHY) layer and media access control (MAC) layer standard because of its lower power consumption characterization. It should be noted that IEEE 802.15.4 implement random back-off of carrier sense multiple access with collision avoidance (CSMA/CA) mechanism to enhance throughput and avoid interference. Although CSMA/CA promotes the quality of wireless communication, its random delay time results in complicated time uncertainty in time synchronization. As a consequence, previous synchronization protocols utilize a great deal of message exchanging to estimate the clock offset and uncertainties [12]. This leads to the network instability especially in the large-scale field such as civil construction sites. Therefore, a new protocol with fast synchronized capabilities and less interference with large network is necessary to be developed.

In this work, we propose to implement the synchronization-on-demand (SOD) protocol for IEEE802.15.4 network. By two data transmission without statistical estimation process, the proposed synchronization with microsecond precision is achieved with minimized resources consumption. To evaluate the functionality of the proposed protocol, furthermore, the ultrasonic localization system is experimentally demonstrated to have the capability to prevent collisions.

\section{Synchronization-On-Demand (SOD) Protocol}

\subsection{Source of Time Uncertainty in WSN}

To obtain the high accurate synchronization within the wireless sensor network, it is important to breakdown the source of uncertainties in the process of radio-frequency (RF) transmission. Among the different categories of time delay factors [15] in "RF transmission”, "interrupt handling time”, "send time”, "receive time”, and "access time" are unpredictable and become the major uncertainties which should be handled in the synchronization process. Each of these unpredictable factors can be understood as follows: "interrupt handling time" is the time interval from MAC layer interrupting the microcontroller to the microcontroller starting to handle package; "send time" is the time required to setup and transfer package to MAC layer in the transmission node; "receive time" is the time needed for handle incoming package in the receiving node; "access time" is the time waiting for RF channel to become clear to transmit. Within these four factors, the “interrupting handling time”, "send time”, and "receive time” are highly depending on the package content. In addition, "interrupting handling time" is also depending on the loading of the microcontroller. And "access time" is depending on the standard specifying the physical and MAC layers for wireless transmissions. It should be noted that "RF transmission" is relative small compared to other factors because the speed of electromagnetic wave propagation is much larger than the transmission distance. Therefore, "RF transmission" is not considered in this work. For WSN based on IEEE 802.15.4 standard, in addition, the "access time" is mainly affected by the uncertain time of random back-off due to CSMA/CA. This time uncertainty dramatically affects the precision of time synchronization.

\subsection{Design of SOD Protocol}

Instead of error estimations, the major design concept of SOD is measuring the time uncertainty information through the time stamping during synchronization procedure. Utilizing this method, nodes can be synchronized with only two transmitted packages from the beacon or specific nodes. Since it can be achieved within extremely short time, it can re-synchronize as requests. As a consequence, the synchronization-on-demand can be implemented without considering clock drifts or skews of the local timer.

To implement the developed synchronization protocol, we assume the crystal oscillator of the beacon node and the receiving node run at the stable frequency. The beacon node sends two packages, synchronization and correction package, to the receiving node. The synchronization package contains the time stamp of the beacon node. And the correction package contains the uncertain time 
information as the beacon sending previous synchronization package. As shown in Fig. 1, the detail time diagram for synchronization procedure can be described as follows: (A) the beacon node reads its time stamp; (B) the time stamp is inserted into synchronization package; (C) the beacon records another time stamp right after sending the synchronization package and the Interval $_{\text {TxSending }}$ can be obtained; (D) the receiver node records its time stamp as finishing receiving package; $(\mathrm{E})$ the Interval ${ }_{\text {TxSending }}$ is inserted into the correction package; (F) the beacon node sends the correction package; $(G)$ the receiver node picks up the correction package and records another time stamp to obtain the Interval ${ }_{\mathrm{RxW} \text { Waiting. }}$ Then the receiver node can calculate the current time stamp of the beacon node as:

$$
\begin{aligned}
\text { Time }_{\text {receiver }}= & \text { Time }_{\text {beacon }}^{\prime} \\
= & \text { Time }_{\text {beacon }}+\text { Interval }_{\text {TxSending }} \\
& + \text { Interval }_{\text {RxWaiting }}+\text { const. }
\end{aligned}
$$

where the "const" term represents the predictable time errors

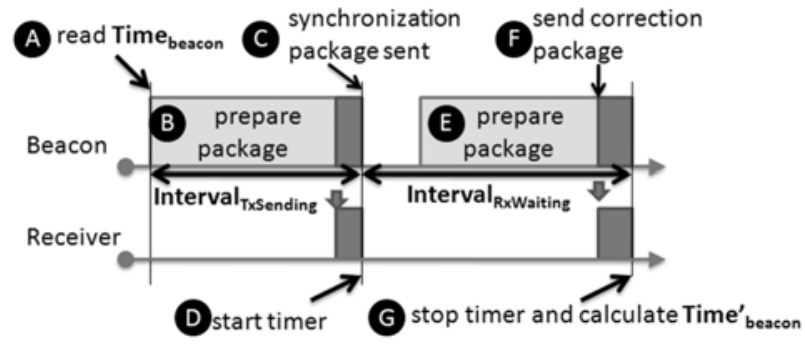

Figure 1. The schematic of the protocol sequence.

Since the microcontroller can record the time as the transmission done, the unpredictable time delays, "Send Time” and "Access Time”, can be measured. Similarly, the "Receive Time" can be obtained. Finally, "Interrupt Handling Time” can be eliminated by firmware coding, such as setting microcontroller into idle state before any transmission during the synchronization procedure. Therefore, most of the unpredictable time delay sources can be identified through the developed SOD protocol. It should be noted that the time stamp exchange is not required in the SOD protocol and it can also be implemented by broadcasting. On the other hand, the
SOD protocol can achieve synchronization with much faster fashion than other statistical-based time synchronization protocol. In addition, the multi-hops synchronization can be achieved by repeating the same procedure to nearby nodes in different regions.

The SOD has several important features that make it suitable for low power wireless sensor network: (A) The high precise clock is only kept at the local beacon. All the other nodes can be aligned up through SOD protocol. (B) It is not necessary for nodes to keep local hardware clock, i.e. crystal oscillator, as statistical methods do. Then nodes can change to low frequency mode to save power as the precise synchronization is not needed. (C) Compared to traditional synchronization methods, the message exchange and iteration are not required in SOD. Moreover, SOD can be applied in CSMA/CA enabled platform like Zigbee, or other wireless network with large access uncertainty.

\subsection{Error Source of SOD}

The developed SOD method measures each time interval directly by hardware timer. Most of the time uncertainties can be eliminated except clock jitter. The clock jitter error can have the following error sources: (A) Cycle $_{R F}$ : jitters between received RF signal input and decoded digital signal output in the RF transceiver chip. This jitter would be one clock cycle of the output block in the RF transceiver chip. (B) Cycle $_{M C U}$ : jitters between the RF transceiver chip and the microcontroller chip. This jitter would be one clock cycle in the microcontroller. (C) Jitters between different nodes. This jitter would be the sum of the (A) and (B) in both nodes. Based on above description, the jitter error between nodes in the same synchronization region will be $4\left(\right.$ Cycle $_{M C U}+$ Cycle $\left._{R F}\right)$. Moreover, in multi-hop topography, the jitter error across synchronization regions can be expressed as $(2+4(n-1))\left(\right.$ Cycle $_{M C U}+$ Cycle $\left._{R F}\right)$, where $n$ is the number of synchronization regions. It should be noted that the jitter error is in Gaussion distribution. Therefore, it can be estimated by Kalman filter.

\subsection{SOD Experimental Verification}


To evaluate the proposed SOD protocol performance and demonstrate its capabilities, we implement the protocol on a low-cost WSN platform for both beacon nodes and receiving nodes. The platform uses UZ2400 and MSP430F1611 as its RF transceiver and microcontroller respectively, as shown in Fig. 2(A) and Fig. 2(B). UZ2400 is IEEE 802.15.4 compatibility RF transceiver [11-12]. It is capable to notify the microcontroller when TX/RX is done. In addition, the MSP430F1611 shares the $5 \mathrm{MHz}$ clock generated from UZ2400. Therefore, there is no jitter appears between these two chips within the platform. In other words, the jitter of Cycle $_{M C U}$ is zero.

(A)

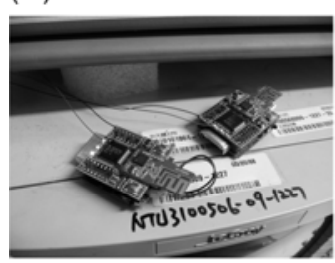

(B)

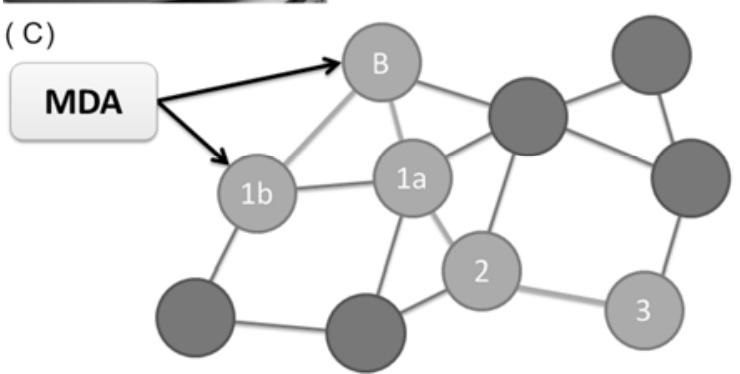

Figure 2. (A) The photo of super nodes; (B) The schematic of the SOD experimental setup for verifications; (C) The schematic of the experimental topography.

To verify the precision of SOD protocol, the experimental network topography can be shown as Fig. 2(C). In this network topography, we adopt three layers to examine the performance of SOD in multi-hop and local synchronization scenarios. Furthermore, we enable CSMA/CA mechanism and follow IEEE 802.15.4 standard during the experiments to mimic the real application scenario. After synchronization, the microcontroller of each node sends the pulse according to the synchronized global time. To identify the synchronized error directly, HP53310A Modulation Domain Analyzer (MDA) is used to analyze the time difference between pulses sent by different nodes. The histogram of synchronization error can be recorded by MDA and shown as Fig. 3. In the experiments with 20,000 measurements for each node, the average synchronization error between the beacon and nodes in one hop is $0.2 \mu$ s with standard deviation of 0.09 $\mu \mathrm{s}$. This error comes from the fundamental clock offset of the $5 \mathrm{MHz}$ crystal. For the nodes in two hops, the mean synchronization error to the beacon is $-0.12 \mu$ s with standard deviation of $0.15 \mu \mathrm{s}$. For the nodes in three hops, the mean synchronization error to the beacon is $-0.17 \mu$ s with standard deviation of $0.2 \mu \mathrm{s}$. Based on these results, the local synchronization precision of the developed SOD protocol can be experimentally determined as $\pm 0.875 \mu \mathrm{s}$ within three hops and the error propagation can be experimentally identified as $0.05 \mu \mathrm{s}$ per hops. This experimental result of clearly demonstrates the high accuracy result can be achieved by SOD without any statistical techniques.
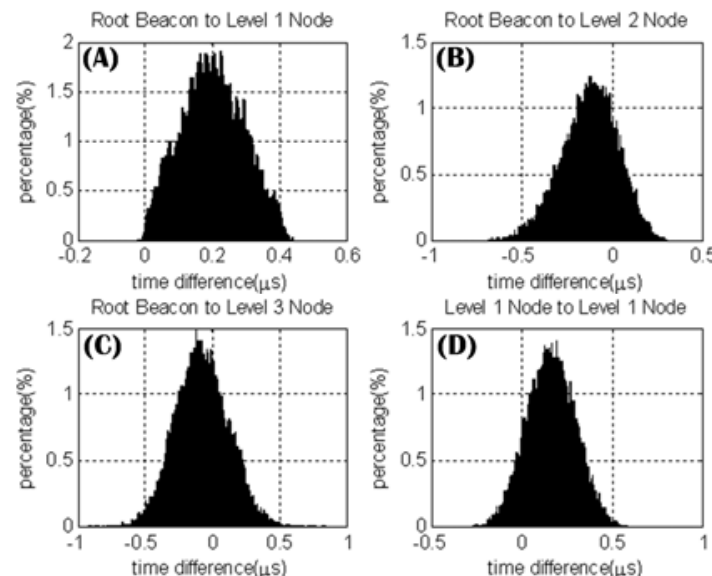

Figure 3. The quantitatively time synchronized results of SOD obtained by Modulation Domain Analyzer. (A) the beacon and nodes are in one-hop range; $(B)$ the beacon and nodes are in two-hop range; $(\mathrm{C})$ the beacon and nodes are in three-hop range; (D) the nodes within one hop. This statistic result demonstrates the synchronization error from single hop to triple hops.

\section{Ultrasonic Localization System}

\subsection{1-D Distance Measurement}

The major advantage of the developed SOD is that it can be achieved specific sensor nodes without global synchronization in the whole network. This dramatically 
improves the network stability and reduces communication latency of the WSN system. To experimentally demonstrate collision-prevention potential of the developed SOD, an ultrasonic ranging system is implemented to measure the distance between two objects. Because of the low cost and ease in implementation, the ultrasonic ranging system utilizes the time-of-flight (TOF) method to obtain the distance information [15-16]. In brief, the TOF method is achieved by two ultrasonic transducers, i.e. sensor nodes. One of them is transmitter and the other is receiver. The transmitter sends out modulated ultrasonic waves. The waves induce the resonant vibration of the transducer in the receiver. The time difference between transmission and receiving can be used to estimate the distance between two transducers. This time difference is achieved by comparing the time stamps from two synchronized ultrasonic sensor nodes. As a consequence, it is critical to have ultra-high precise synchronization.

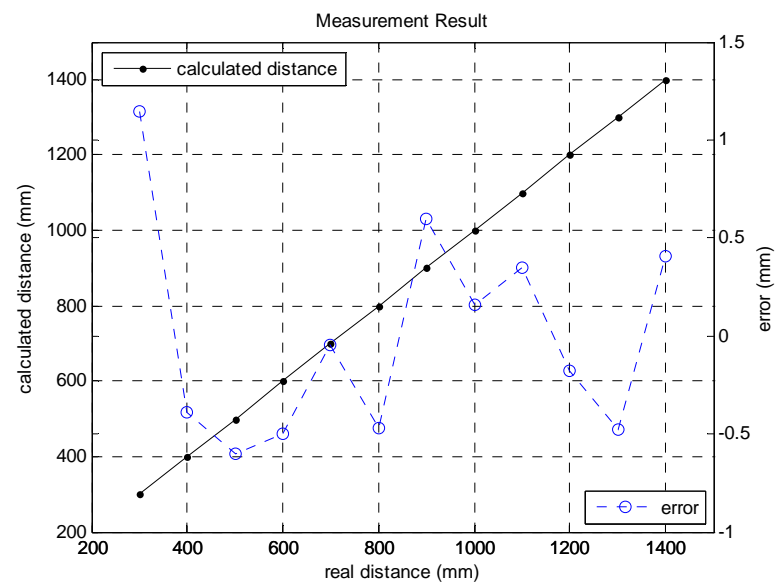

Figure 4. The experimental result of 1-D distance measurement.

In this ultrasonic ranging system, the $40 \mathrm{kHz}$ transducer is used to transmit/receive ultrasonic waves. To quantitatively demonstrate the distance measurement from the developed SOD, only single level synchronization is processed without lost of generalities. For the 1-D distance measurement, the experimental result can be shown as Fig. 4. In Fig.4, it shows the calculated distance and error with regard to real distance. The circle mark stands for error between average and real distances. In general, the error is within $1 \mathrm{~mm}$ at the distance of $2 \mathrm{~m}$.
In other words, the overall standard deviation of synchronization is within 1 us. This result also shows great reproducibility of this ultrasonic distance measurement system.

\subsection{2-D Location Measurement}

The ultrasonic system is reconfigured to process $2 \mathrm{D}$ location measurement. The system diagram is shown as Fig. 5. In Fig. 5, the beacon node sends time synchronization packages every $100 \mathrm{~ms}$. The ultrasonic sender node turns on ultrasonic driver for 100us as synchronized with the beacon node. Because the directivity of ultrasonic transducer is about $\pm 45^{\circ}$, four transducers are arranged to obtain full angle range. The receive sensor node starts to listening ultrasonic signal after synchronized with the beacon node. When the receive sensor node gets ultrasonic signals, it calculates time difference and sends data to the time data collector. Then the time data collector transmits data to computer for analysis. During the experiment, the ultrasonic sender node is put at different grid points. Its location is calculated by the distance information from two receive sensor nodes.
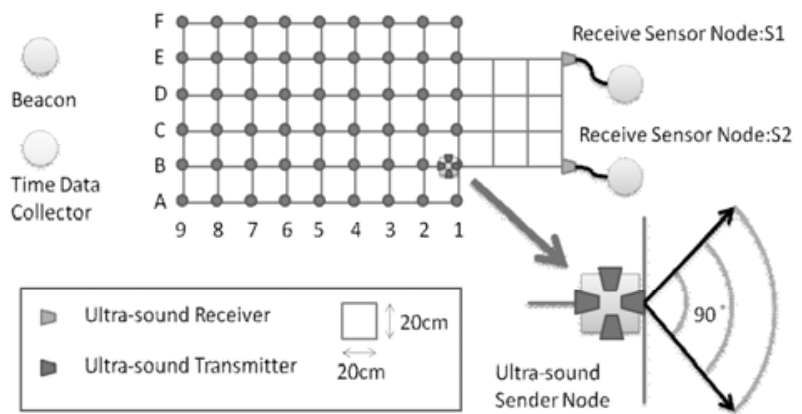

Figure 5. The experimental setup of 2-D distance measurement

To clearly demonstrate capabilities of the ultrasonic ranging system implemented by the developed SOD, the distance errors are labeled in Fig. 6. It is clear that the error is smaller in the center region than in the edge region. This is resulted from the ultrasonic directivity and interference. Based on this experimental result, the maximum location error is $49 \mathrm{~mm}$ within the area of $1 \mathrm{~m}$ by $2 \mathrm{~m}$. 


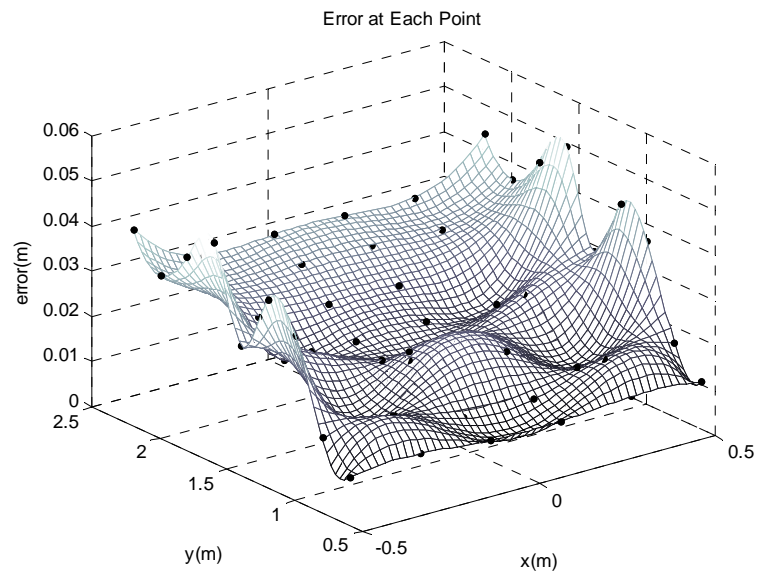

Figure 6. The experimental error of each location in 2D location measurement.

\section{Conclusion}

In summary, we have developed a synchronization-ondemand (SOD) protocol for collision-prevention in civil construction sites. To deal with the large area and complicated movement of equipments, instead of fullnetwork synchronization, the proposed SOD protocol can synchronized within few nodes in $10 \mathrm{~ms}$ with only 0.8 synchronization error. This reduces the wireless communication latency and improves the network stability. In addition, the ultrasonic ranging system with SOD protocol is also experimentally implemented to verify the capability of location identifications. Without complicated estimations and calculations, its location error is less than $5 \mathrm{~cm}$ within $2 \mathrm{~m}$ range. It should be noted that the longer detection range can be achieved by increasing the power of ultrasonic transducers. This work demonstrates a simple and efficient approach to obtain good accuracy, low communication overhead, and robust synchronization for wireless sensor network platforms to be used in automated monitoring in constructions.

\section{Acknowledgement}

This research was financially supported by Nationa l Science Council, Taiwan (Granted Number: NSC 992218-E-002-034-; NSC 99-2218-002-017-).

\section{Reference}

[1] Tserng, H.-P., Ran, B., and Russell, J.-S., "Interactive path planning for multi-equipment landfill operation," Automation in Construction, 10, pp.155-168, 2000.

[2] Oloufa A. A., Ikeada, M., and Oda, Hiroshi, "Situational Awareness of Construction Equipment using GPS, wireless and web technologies," Automation in Construction, 12, pp. 737-748, 2003.

[3] Wu, W., Yang, H., Chew, D.A.S., Yang, S.-H., Gibb, A.G.F., and Li, Q., "Towards An Autonomous Real-Time Tracking System of Near-Miss Accidents on Construction Sites,” Automation in Construction, 19, pp.134-141, 2010.

[4] Chae, S. and Yoshida, T., "Application of RFID Technology to Prevention of Collision Accident with Heavy Equipment," Automation in Construction, 19, pp.368-374, 2010.

[5] Hsieh, Y.-M. and Hung, Y.-C., "A Scalable IT Infrastructure for Automated Monitoring Systems based on The Distributed Computing Technique Using Simple Object Access Protocol Web-Services," Automation in Construction, 18, pp.424-433, 2009.

[6] Milenkovic, A., Otto, C., and Jovanov, E., "Wireless sensor networks for personal health monitoring: Issues and an implementation," Computer Communications, 29, pp. 2521-2533, 2006.

[7] Sheu, J. P., Hu, W. K., and Lin, J. C., "Ratio-based time synchronization protocol in wireless sensor networks," Telecommunication System, 39, pp. 25-35, 2008.

[8] Sadler, B. M., "Local and broadcast clock synchronization in a sensor node," IEEE Signal Processing Letters, 13, pp. 9-12, 2006.

[9] Ganeriwal, S., Popper, C., Capkun, S., and Srivastava, M. B., "Secure time synchronization in sensor networks," ACM Transaction Information and System Security, 11, pp. 23-35, 2008.

[10] Sivrikaya, F. and Yener, B., "Time synchronization in sensor networks: a survey,” IEEE Network, 18, pp. 45-50, 2004.

[11] Rentel, C. H. and Kunz, T. "A mutual network synchronization method for wireless ad hoc and sensor networks," IEEE Transaction Mobile Computing, 7, pp. 633-645, 2008.

[12] Noh, K. Y., Chaudhari, Q. M., Serpedin, E., and Sutter, B. W. "Novel clock phase offset and skew estimation using two-way timing message exchanges for wireless sensor networks," IEEE Transaction Communication, 55, pp. 766777, 2007.

[13] UZ2400 user manual, Uniband Electronic Corporation, Taiwan, Oct. 2007.

[14] IEEE 802.15.4 Specification, 2006 version.

[15] Oriolo, G., Ulivi, G., and Vendittelli, M., "Fuzzy maps: A new tool for mobile robot perception and planning," Journal Robotic System, 14, pp. 179-197, 1997. [16] Tong, F., Tso, S. K., and Xu, T. Z. "A high precision ultrasonic docking system used for automatic guided vehicle," Sensor Actuator A-Phys, 118, pp. 183-189, 2005. 